\title{
Optimal Selection of the Most Reliable Design with a Reciprocal Weibull Degradation Rate
}

\author{
Hong-Fwu Yu \\ Department of Industrial Management, National Formosa University, \\ yuhf@sunws.nfu.edu.tw.
}

\begin{abstract}
In industries, the manufacturer is usually confronted with the problem of selecting the most design among several competing designs for some parts (or components) of his/her product at the research and development stage. Such a selection work is no doubt a great challenge for highly reliable products, since there are few (or even no) failures can be obtained by using traditional life tests or accelerated life tests. In such a case, degradation tests can be employed to assess the product's reliability information if there exists product characteristics whose degradation over time can be related to reliability. Several factors (e.g., the inspection frequency, the sample size and the termination time) are influential to the experimental cost and the selection precision of such selection degradation tests. In this paper, we deal with the optimal design problem of selecting the most reliable design with a reciprocal Weibull degradation rate. First, an intuitively appealing selection rule is proposed. Next, under the constraints of a minimum probability of correct selection and a maximum probability of incorrect selection of the proposed selection rule, the optimum test plan (including the inspection frequency, sample size, and termination time for each competing design) are obtained by minimizing the total experimental cost.
\end{abstract}

Key Words: The most reliable design; Highly reliable products; Inspection frequency; Termination time; Degradation rate; Reciprocal Weibull distribution. 


\section{Introduction}

In industries, the manufacturer usually face the problem of selecting the most reliable design among a variety of competing designs for some parts (or components) of his/her product at the research and development stage. Two techniques are required to achieve such a problem. One is the technique of reliability assessment. The other is a suitable identification rule to select the best design. For the latter, based on complete data, type-II censored data, and randomly censored data, there are several studies (e.g., [5]-[8], [14], [16], [20]) having contributed to this topic. Gupta and Panchapakesan (1988) gave a comprehensive survey of selection procedures in reliability models for complete and/or censored data. For highly-reliable products, Chang, Huang, and Tseng (1992), Tseng, Huang, and Wu (1994), and Tseng (1994) addressed the selection problems under accelerated life tests.

However, for many highly reliable products, it is difficult to assess the reliability of these products using traditional life tests or even using the technique of censoring and/or accelerating the life by testing at higher levels of stress such as elevated temperatures or voltages, because few or even no failures can be obtained in a reasonable amount of time. In such cases, if there exists product characteristics whose degradation over time can be related to reliability, then collecting "degradation data” can provide information about product reliability. Nelson (1990), Bagdonavicius \& Nikulin (2002), and Meeker \& Escobar (1998) surveyed the literature on the subject. Lu \& Meeker (1993) provided a two-stage method which uses degradation data to estimate the failure time distribution. Tseng, Hamada, and Chiao (1995) used a degradation model to improve the fluorescent lamp reliability.

Several factors (e.g., the sample size, the inspection frequency, and the termination time) are influential to the experimental cost and the estimation precision of a degradation test. Obviously, an inappropriate setting of these factors not only wastes the experimental resources, but also reduces the precision of data analysis. Hence, how to set the values of these factors appropriately is usually a concern for the experimenters. Tseng \& Yu (1997) proposed an intuitively on-line real-time rule to determine an appropriate termination time for a degradation experiment. However, they do not address the problem of how to determine the values of all factors simultaneously. Yu \& Tseng (1999) proposed a method to determine the sample size, the inspection frequency, and the termination time for a degradation experiment. Similarly, by using the criterion of minimizing the variance of the estimated $100 p^{\text {th }}$ percentile of a product's lifetime distribution, Wu \& Chang (2002) investigated the optimal combination of these decision variables for a degradation test with the nonlinear mixed-effect model proposed by Lu \& Meeker (1993).

As to the selection problem with degradation data, Yu (2002) proposed an approach to the optimal design of the selection problem for products with lognormal degradation rates. In fact, Weibull and lognormal distributions are much alike and they may fit the lifetime data at hand well in real applications. However, their predictions may lead to a significant difference. An incorrect choice between these two distributions may lead to serious bias. The main purpose of this paper is to deal with the optimal design for a selection problem where the degradation rates follow a reciprocal Weibull distribution. First, an intuitively appealing selection rule is proposed and, then, the optimal test plan is derived by using the criterion of minimizing the total experimental cost. More specifically, subject to a minimum probability of correct selection and a maximum probability of incorrect selection, the optimal combination of the sample size, inspection frequency, and the termination time for each of competing designs are derived such that the total experimental cost is minimal.

The rest of this paper is organized as follows. Section 2 briefly describes the assumptions of a degradation model, the selection rule, and the corresponding optimization problem. Section 3 presents the optimal plan. Section 4 applies the proposed method to a numerical example. Finally, we give a brief conclusion in Section 5.

\section{Assumptions of a Degradation Experiment}

This section is devoted to describing the assumptions of the degradation model, the selection rule, and the optimization problem.

\subsection{The Degradation Model}

Let $\eta(t)=L(t) / L(0) \quad$ denote the standardized quality characteristic of a product at time $t$, where $L(t)$ is the quality characteristic of the product at time $t$. Assume that $\eta(t)$ degrades over time and levels off towards 0 after a period of time. In electronics, this is typical of degradation processes of many highly reliable products (e.g., LEDs, fluorescent lamps, etc.). Based on real applications, assume that $\eta(t)$ satisfies the 
following degradation model:

$$
\phi(\eta(t))=-\beta t^{\alpha}, t \geq 0
$$

where $\phi(x)$ is a non-decreasing function of $x$, defined on $(0,1]$ (e.g., $\phi(x)=\ln x, 1-1 / x$, etc.); $\alpha>0$ is a fixed and known constant; $\beta>0$ is a random variable and is usually called the degradation rate of the product. In practical applications, it isn't easy to determine such a function $\phi(x)$. Yu (2003) gave some explanations about how to select such a function and how to evaluate the appropriateness for the tentatively selected functions. For example, Tseng et al. (1995) used $\phi(x)=\ln x$ with $\alpha=1$ to describe the degradation path of fluorescent lamp. In addition, Yu \& Tseng (1999) and Yu \& Chiao (2000) used $\phi(x)=\ln x \quad$ with $\alpha=0.5$ to describe the degradation paths for LED products.

Let $D$ denote the critical level for this degradation path. The product lifetime $(\tau)$ is suitably defined as the time when $\eta$ crosses the critical level $D$. Then, from Equation (1), $\tau$ can be expressed as

$$
\tau=\left[\frac{-\phi(D)}{\beta}\right]^{1 / \alpha}
$$

In this paper, we assume that $\beta^{-1}$ follows a Weibull distribution with scale parameter $\theta$ and shape parameter $\delta$ (which is denoted by $\beta^{-1} \sim \operatorname{Weibull}(\theta, \delta)$. Then $-\ln \beta$ follows the extreme value distribution with location parameter $u$ and scale parameter $b$, where $u=\ln \theta$ and $b=1 / \delta \quad$ (which is denoted by $-\ln \beta \sim \operatorname{Extreme}(u, b))$. Then it is easily seen that

$$
\ln \tau \sim \text { Extreme }\left(\frac{u+\ln (-\phi(D))}{\alpha}, \frac{b}{\alpha}\right)
$$

\section{Assumptions:}

Suppose that a degradation experiment for selection is conducted under the following conditions:

1. The most reliable design would be selected among $m$ competing designs denoted by $\left\{\Pi_{i}\right\}_{i=1}^{m}$.

2. For each design, $n$ devices are randomly selected for testing.
3. Suppose that, for $\Pi_{i}$, the measurements are made every $f_{i}$ units of time (e.g., $f_{i}$ hours or $f_{i}$ days) until time $t_{i, l_{i}}=f_{i} * l_{i} * t_{u}$, where $t_{u}$ is a unit of time and $l_{i}$ is the number of measurements.

4. Due to the measurement errors, the actual degradation path cannot be observed directly. For $\Pi_{i}$, let $y_{i j}\left(t_{i, k}\right)$ denote the sample degradation path of $j^{\text {th }}$ device at time $t_{i, k}$. It can be expressed as follows:

$$
\begin{gathered}
\phi\left(y_{i j}\left(t_{i, k}\right)\right)=-\beta_{i j} t_{i, k}^{\alpha_{i}}+\varepsilon_{i j}\left(t_{i, k}\right) \\
1 \leq k \leq l_{i}, \quad 1 \leq j \leq n, \quad 1 \leq i \leq m,
\end{gathered}
$$

where $\varepsilon_{i j}\left(t_{i, k}\right)$ is the error term and follows a normal distribution with mean 0 and variance the $\sigma_{\varepsilon}^{2}$ (which is denoted by $N\left(0, \sigma_{\varepsilon}^{2}\right)$ ). Moreover, $\beta_{i j}$ and $\varepsilon_{i j}\left(t_{i, k}\right)$ are independent for all $1 \leq k \leq l_{i}, \quad 1 \leq j \leq n$, and $1 \leq i \leq m$.

5. Assume that $\left\{\alpha_{i}\right\}_{i=1}^{m}$ may be different and that $\beta_{i j}$ follows a reciprocal Weibull distribution with the location parameter $\theta_{i}$ and the scale parameter $\delta$.

6. Let $\tau_{i}$ denote the product's lifetime for $\Pi_{i}$ and $t_{s}$ denote a pre-specified time. Then, according to the assumptions stated above, the probability that a product in $\Pi_{i}$ will be survival beyond $t_{s}$ can be given by

$$
\begin{aligned}
& R_{i}\left(t_{s} ; u_{i}, b\right)=\operatorname{Pr}\left\{\tau_{i} \geq t_{s}\right\} \\
& \quad=1-\Psi\left(\frac{-\left[u_{i}-\alpha_{i} * \ln t_{s}\right]-\ln (-\phi(D))}{b}\right)
\end{aligned}
$$

where $\Psi(x)$ is the cumulative distribution function (cdf) of the standard extreme value distribution. $\Pi_{i^{*}}$ is said to be the most reliable design if $R_{i^{*}}\left(t_{s} ; u_{i^{*}}, b\right)=\max _{1 \leq i \leq m} R_{i}\left(t_{s} ; u_{i}, b\right)$.

According to Equation (4), the equation above implies that $\Pi_{i^{*}}$ is the most reliable design if $u_{i^{*}}-\alpha_{i^{*}} * \ln t_{s}=\max _{1 \leq i \leq m}\left\{u_{i}-\alpha_{i} * \ln t_{s}\right\}$.

Selection rule (SR): 
Let $\left\{\hat{u}_{i}\right\}_{i=1}^{m}$ be unbiased estimators of $\left\{u_{i}\right\}_{i=1}^{m}$ and let $\left(\underline{u}_{i}, \bar{u}_{i}\right)$ be the corresponding $100(1-\zeta) \%$ confidence interval (CI) of $u_{i}$, $1 \leq i \leq m$. Then, based on these estimators, we propose a selection rule as follows:

(SR) $\Pi_{i^{*}}$ is identified to be the most reliable design if

$\underline{u}_{i^{*}}-\alpha_{i^{*}} * \ln t_{s} \geq u_{i}-\alpha_{i} * \ln t_{s}, \quad 1 \leq i \leq m, \quad i \neq i^{*}$

\subsection{The Optimization Problem} follows:

Define the $i^{*}-t h$ preference region as

$\Omega_{i^{*}}=\left\{\vec{u}=\left(u_{1}, u_{2}, \ldots, u_{m}\right) \mid \underline{u}_{i^{*}}-\alpha_{i^{*}} * \ln t_{s} \geq u_{i}-\alpha_{i} * \ln t_{s}+\Delta, 1 \leq i \leq m, i \neq i^{*}\right\}$

where $\Delta>0$ is a constant pre-specified by the decision maker. We say that Rule SR gives a correct decision (CD) for $\vec{u}=\left(u_{1}, u_{2}, \ldots, u_{m}\right) \in \Omega_{i^{*}}$, if $\Pi_{i^{*}}$ is identified as the most reliable design. Let $\operatorname{Pr}_{\vec{u}}(\mathrm{CD} \mid \mathrm{SR})$ and $\operatorname{Pr}_{\vec{u}}(\mathrm{ICD} \mid \mathrm{SR})$ denote the probabilities that Rule SR gives a correct decision and an incorrect decision for $\vec{u}$, respectively. To enhance the quality of our decision, it is usually required that the probability of $\mathrm{CD}$ exceeds a specified minimum value $P^{*}$ (referred to as the $P^{*}$-condition) and the probability of ICD is less than a guarantee of maximum value $\xi^{*}$ (referred to as the $\xi^{*}$-condition); that is,

$$
\underset{\vec{u} \in \Omega_{i^{*}}}{\inf } \operatorname{Pr}_{\vec{u}}(\mathrm{CD} \mid \mathrm{SR}) \geq P^{*}
$$

and

$$
\sup _{\vec{u} \in \Omega_{i^{*}}} \operatorname{Pr}_{\vec{u}}(\mathrm{ICD} \mid \mathrm{SR}) \leq \xi^{*}
$$

where $P^{*}$ and $\xi^{*}$ are pre-determined values given by the decision maker. Obviously, these two conditions will lead to several combinations of decision variables $\left(\left\{\left(f_{i}, l_{i}\right)\right\}_{i=1}^{m}, n\right)$, which are closely related to the experimental cost. Due to the limitation of experimental resources, the manufacturer usually wishes to control the experimental cost as low as possible. Thus, a trade-off is needed. Let $\operatorname{TC}\left(\left\{\left(f_{i}, l_{i}\right)\right\}_{i=1}^{m}, n\right)$ denote the total cost of conducting the degradation experiment. Then a typical decision problem can be formulated as follows:

$$
\begin{aligned}
& \text { Minimize } \operatorname{TC}\left(\left\{\left(f_{i}, l_{i}\right)\right\}_{i=1}^{m}, n\right) \\
& \text { Subject to } \\
& \inf _{\vec{u} \in \Omega_{i^{*}}} \operatorname{Pr}_{\vec{u}}(\mathrm{CD} \mid \mathrm{SR}) \geq P^{*} \\
& \sup _{\vec{u} \in \Omega_{i^{*}}} \operatorname{Pr}_{\vec{u}}(\mathrm{ICD} \mid \mathrm{SR}) \leq \xi^{*} \\
& f_{i}, \quad l_{i}, \quad n \in \mathbf{N}=\{1,2,3, \ldots\} \\
& i=1,2, \ldots, \quad m .
\end{aligned}
$$

\section{The Optimal Plan}

The framework for solving the optimization model consists of three major steps stated in Sections 3.1-3.3.

\subsection{The Estimation of $\left(\left\{u_{i}\right\}_{i=1}^{m}, b\right)$}

For $1 \leq j \leq n$ and $1 \leq i \leq m$, based on the observations $\left\{\left(t_{i, k}, y_{i j}\left(t_{i, k}\right)\right)\right\}_{k=1}^{l_{i}}$, the least-squares estimator (LSE) $\hat{\beta}_{i j}$ of $\beta_{i j}$, conditional on $\beta_{i j}$, can be computed by minimizing

$$
L S\left(\beta_{i j}\right)=\sum_{k=1}^{l_{i}}\left[\phi\left(y_{i j}\left(t_{i, k}\right)\right)+\beta_{i j} \alpha_{i, k}^{\alpha_{i}}\right]^{2}
$$

Thus, we obtain

$$
\hat{\beta}_{i j}=-\frac{\sum_{k=1}^{l_{i}} \phi\left(y_{i j}\left(t_{i, k}\right)\right) t_{i, k}^{\alpha_{i}}}{\sum_{k=1}^{l_{i}} t_{i, k}^{2 \alpha_{i}}}
$$

and $\sigma_{\varepsilon}^{2}$ can be estimated by

$$
\sigma_{\varepsilon}^{2}=\sum_{i=1}^{m} \sum_{j=1}^{n} \frac{1}{m n\left(l_{i}-1\right)} L S\left(\hat{\beta}_{i j}\right)
$$

According to Yu \& Tseng (2004), if $\sum_{k=1}^{l_{i}} t_{i, k}^{2 \alpha_{i}}$ is sufficiently large, then $-\ln \hat{\beta}_{i j}$ approximately 
follows the following extreme value distribution:

$$
-\ln \hat{\beta}_{i j} \sim \operatorname{Extreme}\left(u_{i(l)}, b_{i(l)}\right)
$$

Where

$$
\begin{aligned}
& u_{i(l)} \approx u_{i}+\gamma *\left(b_{i(l)}-b\right) \\
& b_{i(l)}=\left[b^{2}+\frac{6 \sigma_{\varepsilon}^{2} \theta_{i}^{2} \Gamma\left(1+\frac{2}{\delta}\right)}{\pi^{2} \sum_{k=1}^{l_{i}} t_{i, k}^{2 \alpha_{i}}}\right]^{1 / 2}
\end{aligned}
$$

$\gamma=0.5772 \ldots$ is known as Euler's constant, and $\Gamma(x)$ is the gamma function. Based on $\left\{-\ln \hat{\beta}_{i j}\right\}_{j=1}^{n}$, Yu \& Tseng (2004) estimated $u_{i}$ and $b$ by $\hat{u}_{i}$ and $\hat{b}_{i}$ and which satisfy the following simultaneous equations:

$$
e^{\hat{u}_{i}}=\left[\frac{1}{n} \sum_{j=1}^{n} \exp \left(\frac{x_{i j}}{\hat{b}_{i}}\right)\right]^{\hat{b}_{i}}
$$

and

$$
\frac{\sum_{j=1}^{n} x_{i j} \exp \left(\frac{x_{i j}}{\hat{b}_{i}}\right)}{\sum_{j=1}^{n} \exp \left(\frac{x_{i j}}{\hat{b}_{i}}\right)}-\hat{b}_{i}-\frac{1}{n} \sum_{j=1}^{n} x_{i j}=0,
$$

where $x_{i j}=-\ln \hat{\beta}_{i j}, 1 \leq j \leq n$ and $1 \leq i \leq m$. Note that $\hat{u}_{i}$ and $\hat{b}_{i}$ are the maximum likelihood estimators (MLEs) of $u_{i(l)}$ and $b_{i(l)}$ based on $\left\{-\ln \hat{\beta}_{i j}\right\}_{j=1}^{n}$.

\subsection{The Sampling Distributions of}

$$
\left(\left\{\hat{u}_{i}\right\}_{i=1}^{m}, \hat{b}_{i}\right)
$$

According to Yu \& Tseng (2004), if $\sum_{k=1}^{l_{i}} t_{i, k}^{2 \alpha_{i}}$ is sufficiently large, then the joint distribution of $\hat{u}_{i}$ and $\hat{b}_{i}$ follows asymptotically the following bivariate normal distribution:

$$
\left(\begin{array}{l}
\hat{u}_{i} \\
\hat{b}_{i}
\end{array}\right) \sim N\left(\left(\begin{array}{l}
u_{i(l)} \\
b_{i(l)}
\end{array}\right), \Sigma_{i(l)}\right)
$$

where

$$
\Sigma_{i(l)}=\left[\begin{array}{cc}
\frac{6 b_{i(l)}^{2}}{n \pi^{2}} *\left[\frac{\pi^{2}}{6}+(1-\gamma)^{2}\right. & \frac{6 b_{i(l)}^{2}}{n \pi^{2}} *(\gamma-1) \\
\frac{6 b_{i(l)}^{2}}{n \pi^{2}} *(\gamma-1) & \frac{6 b_{i(l)}^{2}}{n \pi^{2}}
\end{array}\right]
$$

Furthermore, as $\sum_{k=1}^{l_{i}} t_{i, k}^{2 \alpha_{i}}$ approaches to infinity, $N\left(\left(\begin{array}{l}u_{i(l)} \\ b_{i(l)}\end{array}\right), \Sigma_{i(l)}\right)$ will converge to $N\left(\left(\begin{array}{c}u_{i} \\ b\end{array}\right), \Sigma\right)$, where

$$
\Sigma=\left[\begin{array}{cc}
\frac{6 b^{2}}{n \pi^{2}} *\left[\frac{\pi^{2}}{6}+(1-\gamma)^{2}\right. & \frac{6 b^{2}}{n \pi^{2}} *(\gamma-1) \\
\frac{6 b^{2}}{n \pi^{2}} *(\gamma-1) & \frac{6 b^{2}}{n \pi^{2}}
\end{array}\right]
$$

Adopting $\Sigma$ as a benchmark, Yu (2004) used the relative error of $\Sigma_{i(l)}$ and $\Sigma$ defined by $v_{r}^{i}=\frac{\left\|\Sigma_{i(l)}-\Sigma\right\|_{\infty}}{\|\Sigma\|_{\infty}}$ as a measure to assess the size of the difference between $\left(\begin{array}{l}u_{i(l)} \\ b_{i(l)}\end{array}\right)$ and $\left(\begin{array}{c}u_{i} \\ b\end{array}\right)$ (and $\Sigma_{i(l)}$ and $\Sigma$ ), and then to determine if $\sum_{k=1}^{l_{i}} t_{i, k}^{2 \alpha_{i}}$ is sufficiently large, where the $\infty$-norm $\|\cdot\|_{\infty}$ for an $m \times n$ matrix $A=\left[a_{i j}\right]$ is defined as follows (see Golub \& Van Loan, 1989):

$$
\|A\|_{\infty}=\max _{1 \leq i \leq n} \sum_{j=1}^{m}\left|a_{i j}\right|
$$

In this paper, we also set the following condition proposed by $\mathrm{Yu}$ (2004) to choose $\left\{\left(f_{i}, l_{i}\right)\right\}_{i=1}^{m}$ :

$$
v_{r}^{i}=\rho, 1 \leq i \leq m
$$


where $\rho>0$ is a constant pre-specified by the decision maker. This condition leads to the following result:

\section{Proposition 1.}

$$
\frac{6 \sigma_{\varepsilon}^{2} \theta_{i}^{2} \Gamma\left(1+\frac{2}{\delta}\right)}{\pi^{2} \sum_{k=1}^{l_{i}} t_{i, k}^{2 \alpha_{i}}}=b^{2} \rho, 1 \leq i \leq m
$$

Equation (11) implies that $b_{i(l)}$ 's are approximately equal. For convenience, we set

$$
b_{i(l)}=b_{(l)}=b * \sqrt{1+\rho}, 1 \leq i \leq m
$$

Then

$$
\begin{aligned}
& \operatorname{Var}\left[\hat{u}_{i}\right]=\sigma_{u}^{2}=\frac{6 b_{(l)}^{2}}{n \pi^{2}} *\left[\frac{\pi^{2}}{6}+(1-\gamma)^{2}\right], \\
& 1 \leq i \leq m, \\
& \operatorname{Var}\left[\hat{b}_{i}\right]=\frac{6 b_{(l)}^{2}}{n \pi^{2}}, 1 \leq i \leq m, \\
& \operatorname{Cov}\left[\hat{u}_{i}, \hat{b}_{i}\right]=\frac{6 b_{(l)}^{2}}{n \pi^{2}} *(\gamma-1), 1 \leq i \leq m,
\end{aligned}
$$

and

$$
u_{i(l)}=u_{i}+\gamma *\left(b_{(l)}-b\right), 1 \leq i \leq m .
$$

\subsection{The Computation of $\left(\underline{u}_{i}, \bar{u}_{i}\right)$}

Due to mathematical and computational intractability, it is usually a problem for obtaining CI for the location parameter of an extreme value distribution (Lawless, 1982). A feasible way to do this is based on the following pivotal:

$$
Z_{u_{i}}=\frac{\hat{u}_{i}-u_{i}}{\hat{b}_{i}}, 1 \leq i \leq m .
$$

To prove $Z_{u_{i}}$ 's are pivotals, we need the following result (see Lawless, 1982):

Lemma 1. Based on Equation (9), $Z_{1_{i}}=\frac{\hat{u}_{i}-u_{i(l)}}{\hat{b}_{i}}$ and $Z_{2_{i}}=\frac{\hat{b}_{i}}{b_{i(l)}}$ are pivotals.
Subsequently, according to Lemma 1 and Equation (12), the fact that $Z_{u_{i}}$ is a pivotal can be easily shown by the following manipulations:

$$
\begin{aligned}
& \frac{\hat{u}_{i}-u_{i}}{\hat{b}_{i}} \\
& =\frac{\hat{u}_{i}-\left[u_{i}+\gamma *\left(b_{i(l)}-b\right)\right]+\gamma *\left(b_{i(l)}-b\right)}{\hat{b}_{i}} \\
& =Z_{1_{i}}+\frac{b_{(l)} \gamma *\left(1-\frac{1}{\sqrt{1+\rho}}\right)}{\hat{b}_{i}} \\
& =Z_{1_{i}}+\gamma *\left(1-\frac{1}{\sqrt{1+\rho}}\right) \frac{1}{Z_{2_{i}}}
\end{aligned}
$$

Although CIs for $u_{i}$ can in principle be obtained from $Z_{u_{i}}$, a practical difficulty is that its distribution can be very complicated. Hence, it is impossible to obtain by analytical means exact percentage points for it (see Lawless, 1982). An alternative to dissolve this difficulty is to produce very close estimates of percentage points by Monte Carlo methods. This is because that $Z_{1_{i}}$ and $Z_{2_{i}}$ are pivotals (i.e., parameter-free), their distributions are the same irrespective of the values of $u_{i(l)}$ and $b_{(l)}$. So, if we set $u_{i(l)}=0$ and $b_{(l)}=1$, then $Z_{1_{i}}$ and $Z_{2_{i}}$ become

$$
Z_{1_{i}}=\frac{\hat{u}_{i}}{\hat{b}_{i}} \text { and } Z_{2_{i}}=\hat{b}_{i} .
$$

Also, the right-hand side of the last equation in the expression for $\frac{\hat{u}_{i}-u_{i}}{\hat{b}_{i}}$ above can be reduced as follows:

$$
\frac{\hat{u}_{i}}{\hat{b}_{i}}+\gamma\left(1-\frac{1}{\sqrt{1+\rho}}\right) * \frac{1}{\hat{b}_{i}} .
$$

Thus, a very good estimate of the distribution of $Z_{u_{i}}$ can be obtained by generating many (e.g., 20000) samples from the standard extreme value distribution, computing $\hat{u}_{i}$ and $\hat{b}_{i}$, and obtaining 
the values of $\hat{u}_{i} / \hat{b}_{i}$ and $1 / \hat{b}_{i}$. In this paper, we provide a table for $Z_{u_{i}}$ for $15 \leq n \leq 45$ with percentage points $(0.005,0.025,0.05,0.95,0.975$, 0.995). For each combination of $n$ and percentage point, 20000 samples are generated from the standard extreme value distribution by S-plus package. The results are listed in Table 1.

For illustrative purposes, based on such simulation results, a two-sided CI for $u_{i}$ can be obtained as follows. Suppose that we want a two-sided $100(1-\zeta) \% \mathrm{CI}$ for $u_{i}$ and suppose that, from Table 1, we find that

$$
\operatorname{Pr}\left\{\omega_{1} \leq Z_{u_{i}} \leq \omega_{2}\right\}=1-\zeta,
$$

where $\omega_{1}=\omega_{1}(n, \zeta)$ and $\omega_{2}=\omega_{2}(n, \zeta)$ denote the $100(1-\zeta) \%$ lower and upper confidence limits for $Z_{u_{i}}$ with sample size $n$, respectively. This gives the $\mathrm{CI}$ for $u_{i}$ as follows:

$$
\underline{u}_{i} \leq u_{i} \leq \bar{u}_{i}
$$

where $\underline{u}_{i}=\hat{u}_{i}-\omega_{2} \hat{b}_{i}$ and $\bar{u}_{i}=\hat{u}_{i}-\omega_{1} \hat{b}_{i}$.

3.4 The Computation of $\inf _{\vec{u} \in \Omega_{i^{*}}} \operatorname{Pr}_{\vec{u}}(\mathrm{CD} \mid \mathrm{SR})$

$$
\text { and } \sup _{\vec{u} \in \Omega_{i^{*}}} \operatorname{Pr}_{\vec{u}}(\mathrm{ICD} \mid \mathrm{SR})
$$

According to Equations (5), (6), (12)-(15), and (16), we can obtain the following result:

\section{Proposition 2.}

$$
\begin{aligned}
& \underset{\vec{u} \in \Omega_{i^{*}}}{\operatorname{Pr}_{\vec{u}}(\mathrm{CD} \mid \mathrm{SR})} \\
& =\int_{0}^{1}\left\{\Phi\left[\Phi^{-1}(x) * \frac{\sigma_{2}}{\sigma_{1}}+\frac{\Delta+\left(\omega_{1}-\omega_{2}\right) b \sqrt{1+\rho}}{\sigma_{1}}\right]\right\}^{m-1} d x
\end{aligned}
$$

and

$$
\begin{aligned}
& \sup _{\vec{u} \in \Omega_{i^{*}}} \operatorname{Pr}_{\vec{u}}(\mathrm{ICD} \mid \mathrm{SR}) \\
& =1-\int_{0}^{1}\left\{\Phi\left[\Phi^{-1}(x) * \frac{\sigma_{1}}{\sigma_{2}}+\frac{\Delta+\left(\omega_{2}-\omega_{1}\right) b \sqrt{1+\rho}}{\sigma_{2}}\right]\right\}^{m-1} d x
\end{aligned}
$$

where

$$
\sigma_{1}^{2}=\frac{6 b_{(l)}^{2}}{n \pi^{2}} *\left[\frac{\pi^{2}}{6}+(1-\gamma)^{2}+\omega_{1}^{2}+2 \omega_{1}(1-\gamma)\right]
$$

$$
\sigma_{2}^{2}=\frac{6 b_{(l)}^{2}}{n \pi^{2}} *\left[\frac{\pi^{2}}{6}+(1-\gamma)^{2}+\omega_{2}^{2}+2 \omega_{2}(1-\gamma)\right]
$$

and $\Phi(x)$ is the cdf of the standard normal distribution.

\subsection{The Characterization of}

$$
\mathbf{T C}\left(\left\{\left(f_{i}, l_{i}\right)\right\}_{i=1}^{m}, n\right)
$$

The total cost of experiment, $\mathrm{TC}\left(\left\{\left(f_{i}, l_{i}\right)\right\}_{i=1}^{m}, n\right)$, consists of three parts:

1. The cost of conducting the experiment is $C_{s} * \max _{1 \leq i \leq m}\left\{f_{i} * l_{i}\right\}+C_{p} * \sum_{i=1}^{m} f_{i} * l_{i}$, where $C_{s}$ denotes the operator's salary per unit of time and $C_{p}$ denotes the unit cost of power bill and depreciation of the currency of testing equipment.

2. The cost of measurement is $C_{m} * n * \sum_{i=1}^{m} l_{i}$, where $C_{m}$ denotes the unit cost of measurement.

3. The cost of tested devices is $C_{d} * m * n$, where $C_{d}$ denotes the unit cost of device.

Thus, the total cost of experiment is

$$
\begin{aligned}
& \operatorname{TC}\left(\left\{\left(f_{i}, l_{i}\right)\right\}_{i=1}^{m}, n\right) \\
& =C_{s} * \max _{1 \leq i \leq m}\left\{f_{i} * l_{i}\right\}+C_{p} * \sum_{i=1}^{m} f_{i} * l_{i} \\
& \quad+C_{m} * n * \sum_{i=1}^{m} l_{i}+C_{d} * m * n .
\end{aligned}
$$

\section{The optimization model:}

Synthesizing the results above, the optimization model can be expressed as follows:

\section{Minimize}

$$
C_{s} * \max _{1 \leq i \leq m}\left\{f_{i} * l_{i}\right\}+C_{p} * \sum_{i=1}^{m} f_{i} * l_{i}+
$$

$$
C_{m} * n * \sum_{i=1}^{m} l_{i}+C_{d} * m * n
$$

\section{Subject to}




$$
\begin{aligned}
& \int_{0}^{1}\left\{\Phi\left[\Phi^{-1}(x) * \frac{\sigma_{2}}{\sigma_{1}}+\frac{\Delta+\left(\omega_{1}-\omega_{2}\right) b \sqrt{1+\rho}}{\sigma_{1}}\right]\right\}^{m-1} d x \geq P^{*} \\
& \int_{0}^{1}\left\{\Phi\left[\Phi^{-1}(x) * \frac{\sigma_{1}}{\sigma_{2}}+\frac{\Delta+\left(\omega_{2}-\omega_{1}\right) b \sqrt{1+\rho}}{\sigma_{2}}\right]\right\}^{m-1} d x \geq 1-\xi^{*}
\end{aligned}
$$

$$
\begin{aligned}
& \frac{6 \sigma_{\varepsilon}^{2} \theta_{i}^{2} \Gamma\left(1+\frac{2}{\delta}\right)}{\pi^{2} \sum_{k=1}^{l_{i}} t_{i, k}^{2 \alpha_{i}}}=b^{2} \rho \\
& f_{i}, \quad l_{i}, \quad n \in \mathbf{N}=\{1,2,3, \ldots\} n \geq 2 \\
& i=1,2, \ldots, \quad m .
\end{aligned}
$$

Although the optimization model seems somewhat complicated, a close look at the constraints reveals that $\left\{\left(f_{i}, l_{i}\right)\right\}_{i=1}^{m}$ can be determined by Equations (11) and $n^{*}$ can be determined by Equations (17) and (18) which are really functions of $n$.

In the next section, we will use an example to illustrate this procedure.

\section{A Numerical Example}

Suppose that a manufacturer wants to select the most reliable design at a predetermined time $t_{s}=20000$ hours among four competing designs of parts (denoted by $\left\{\Pi_{i}\right\}_{i=1}^{4}$ ) whose quality characteristics satisfy Equation (3) with $\phi(x)=\ln x$ and $\beta_{i j}^{-1} \sim \operatorname{Weibull}\left(\theta_{i}, \delta\right)$ (i.e., $\left.-\ln \beta_{i j} \sim \operatorname{Extreme}\left(u_{i}, b\right)\right)$. If the manufacturer would like to conduct degradation experiments to select the optimal design and would like to control the quality of decision such that the probability of correct selection achieves 0.90, then the following questions may come to him:

1. How many devices $(n)$ should be taken for each design?

2. How to determine an appropriate inspection frequency $\left(f_{i}\right)$ for $\Pi_{i}$ ?

3. How many times $\left(l_{i}\right)$ should the measurements be made for $\Pi_{i}$ ? (In other words, what is the most appropriate termination time $\left(t_{i, l_{i}}\right)$ for $\Pi_{i}$ ?)

To answer these questions, he needs the values of $\left\{u_{i}\right\}_{i=1}^{4}, b$, and $\sigma_{\varepsilon}^{2}$. So a pilot study is conducted as follows. For each competing design, $n_{0}$ devices are randomly selected for performing a degradation test under the condition that the measurements are made every $f_{0}$ units of time until time $t_{l_{0}}=f_{0} * l_{0} * t_{u}$.

Suppose, based on the data obtained from the pilot study and the procedures in Section 3, that $\left(\alpha_{1}, \alpha_{2}, \alpha_{3}, \alpha_{4}\right)=(0.5,0.5,0.5,0.5)$ and

$$
\begin{aligned}
& \left(u_{1}, u_{2}, u_{3}, u_{4}, b, \sigma_{\varepsilon}^{2}\right) \\
& \cong(5.1980,5.0438,4.8075,4.6923,0.120,0.0020) .
\end{aligned}
$$

According to Equation (19), we can take $\Delta=5.1980-5.0438=0.1542$. Besides, set $\zeta=0.10$, $\rho=0.01, \quad P^{*}=0.90, t_{s}=20000$ hours, $t_{u}=24$ hours, and

$$
\left(C_{s}, C_{p}, C_{m}, C_{d}\right)=(18.25,10.85,1.25,60) .
$$

Finally, if the lifetimes of these designs are technically defined as the time when their quality characteristics degrade below a critical level $D=50 \%$, then the optimal test plan can be obtained as follows:

$$
\left(f_{1}^{*}, f_{2}^{*}, f_{3}^{*}, f_{4}^{*}, l_{1}^{*}, l_{2}^{*}, l_{3}^{*}, l_{4}^{*}, n^{*}\right)
$$$$
=(2,2,3,3,101,87,56,49,25) \text {. }
$$

That is, there are 25 devices on test for each design. And, the inspection for $\Pi_{1}, \Pi_{2}, \Pi_{3}$, and $\Pi_{4}$ will be taken up to $t_{1, l_{1}^{*}}=2 * 101 * 24=4848$, $t_{2, l_{2}^{*}}=2 * 87 * 24=4176, \quad t_{3, l_{3}^{*}}=3 * 56 * 24=4032$, and $t_{4, l_{4}^{*}}=3 * 49 * 24=3528$ hours at $48,48,72$, and 72 hour intervals, respectively.

The total cost is $\operatorname{TC}\left(\left\{\left(f_{i}^{*}, l_{i}^{*}\right)\right\}_{i=1}^{m}, n^{*}\right)=26340.1$ dollars.

Thus, by using the selecting rule SR, we have at least $90 \%$ confidence in selecting the most reliable design correctly, if the true configuration of $\left(u_{1}, u_{2}, u_{3}, u_{4}, b, \sigma_{\varepsilon}^{2}\right)$ is as shown above. 


\section{Conclusion}

This paper proposed an approach to the optimal design problem of selecting the most reliable design with a reciprocal Weibull degradation rate. First, an intuitively appealing selection rule is proposed. Then the optimal combination of the sample size, inspection frequency, and the termination time for each of competing products is derived by minimizing the total experimental cost, subject to the constraints of a minimum probability of correct selection and a maximum probability of incorrect selection of the proposed selection rule.

For some very-highly-reliable products, the degradation may be so slow that it is impossible to have a precise estimation within a reasonable amount of testing time. In such cases, an alternative is to use higher stresses to extrapolate the products' reliability at a design stress. This is called an accelerated degradation test (ADT). Many excellent references can be found in Nelson (1990), Bagdonavicius \& Nikulin (2002), and Meeker \& Escobar (1993) on this subject. It is no doubt interesting to explore the selection problems with ADT data.

\section{References}

[1] Bagdonavicius, V. and Nikulin, M., 2002, Accelerated Life Models: modeling and statistical analysis, Chapman \& Hall/CRC, New York.

[2] Chang, D. S., Huang, D. Y. and Tseng, S. T., 1992, Selecting the most reliable design under type-II censored accelerated test, IEEE Transactions on Reliability, 41, 588-592.

[3] Golub, G. H. and Van Loan, C. F., 1989, Matrix Computations, 2nd edition, Johns Hopkins, Baltimore.

[4] Gupta, S. S. and Panchapakesan, S., 1988, Selection and ranking procedures in reliability models, Handbooks of Statistics, 7 (P. R. Krishnaiah and C. R. Rao, Eds.), North-Holland, Amsterdam, 131-156.

[5] Kim, J. S., 1988, A subset selection procedure for exponential populations under random censoring, Communications in Statistics Theory, Methods, 17, 183-206.

[6] Kingston, J. V. and Patel, J. K., 1980a, Selecting the best one of several Weibull populations, Communications in Statistics - Theory, Method, 9, 383-398.

[7] Kingston, J. V. and Patel, J. K., 1980b, A restriction subset selection procedure for Weibull populations, Communications in Statistics - Theory, Method, 9, 1371-1383.
[8] Kingston, J. V. and Patel, J. K., 1982, Classifying Weibull populations with respect to a control, Communications in Statistics - Theory, Method, 11, 899-909.

[9] Lawless, J. F., 1982, Statistical Models and Methods for Lifetime Data, John Wiley \& Sons, New York.

[10] Lu, C. J. and Meeker, W. Q., 1993, Using degradation measures to estimate a time-to-failure distribution, Technometrics, 35, 161-174.

[11] Meeker, W. Q. and Escobar, L. A., 1993, A review of research and current issues in accelerated testing, International Statistical Review, 61, 147-168.

[12] Meeker, W. Q. and Escobar, L. A., 1998, Statistical Methods for Reliability Data, John Wiley \& Sons, New York.

[13] Nelson, W., 1990, Accelerated Testing: statistical models, test plans, and data analysis, John Wiley \& Sons, New York.

[14] Tseng, S. T., 1988, Selecting more reliable Weibull populations than a control, Communications in Statistics - Theory, Method, 17, 169-180.

[15] Tseng, S. T., 1994, Planning accelerated life tests for selecting the most reliable product under the Weibull-inverse power model, Journal of Statistical Planning and Inference, 41, 215-230.

[16] Tseng, S. T. and Chang, D. S., 1989, Selecting the most reliable design under type-II censoring, Reliability Engineering and System Safety, 25, 147-156.

[17] Tseng, S. T., Hamada, M. and Chiao, C. H., 1995, Using degradation data to improve fluorescent lamp reliability, Journal of Quality Technology, 27, 363-369.

[18] Tseng, S. T., Huang, D. Y. and Wu, T. Y., 1994, A sampling plan for selecting the most reliable product under the Arrhenius accelerated life test model, Statistica Sinica, 4, 233-247.

[19] Tseng, S. T. and Yu, H. F., 1997, A termination rule for degradation experiments, IEEE Transactions on Reliability, 46, 130-133.

[20] Tseng, S. T. and Wu, H. J., 1990, Selecting, under type-II censoring, Weibull populations that are most reliable, IEEE Transactions on Reliability, 39, 193-198.

[21] Yu, H. F., 2002, Optimal selection of the most reliable product with degradation data, Engineering Optimization, 34, 579-590.

[22] Yu, H. F., 2003, Designing an accelerated degradation experiment by optimizing the estimation of the percentile, Quality and Reliability Engineering International, 19 (3), 
197-214.

[23] Yu, H. F., 2004, Designing an accelerated degradation experiment with a reciprocal Weibull degradation rate. To appear in Journal of Statistical Planning and Inference.

[24] Yu, H. F. and Chiao, C. H., 2000, Designing a degradation experiment by optimizing the interval estimation of the percentile, Industrial Engineering Research, 2, 31-46.

[25] Yu, H. F. and Tseng, S. T., 1999, Designing a degradation experiment, Naval Research Logistics, 46, 689-706.

[26] Yu, H. F. and Tseng, S. T., 2004, Designing a degradation experiment with a reciprocal Weibull degradation rate, Quality Technology and Quantitative Management, 1 (1), 47-63.

[27] Wu, S. J. and Chang, C. T., 2002, Optimal design of degradation tests in presence of cost constraint, Reliability Engineering \& System Safety, 76, 109-115. 
Table 1. Percentage points for $Z_{u_{i}}$ with $15 \leq n \leq 45$

\begin{tabular}{|c|c|c|c|c|c|c|}
\hline \multirow[b]{2}{*}{$n$} & \multicolumn{6}{|c|}{ Percent } \\
\hline & 0.005 & 0.025 & 0.05 & 0.95 & 0.975 & 95 \\
\hline 15 & .8369349 & .613270 & .50511 & 1999568 & 149584 & 64780 \\
\hline 16 & 0.8063064 & -0.5696472 & -0.4676821 & 0.4857601 & .5996278 & 8321308 \\
\hline 17 & .7716773 & -0.5652294 & -0.4626510 & 0.4681919 & 5810484 & 7919772 \\
\hline 18 & .7593111 & -0.5446783 & -0.4408019 & .4496273 & .5431534 & .7716725 \\
\hline 19 & .7200131 & -0.5221917 & -0.4349377 & 0.4389673 & 5317767 & 0.7608980 \\
\hline 20 & .6992567 & -0.5124626 & 47361 & 267159 & 5185241 & .7403819 \\
\hline 21 & .6746095 & -0.5000683 & -0.4108292 & 0.4150886 & 5036729 & .6956545 \\
\hline 22 & .6461502 & -0.4746067 & 63549 & 335923 & 786128 & 6742980 \\
\hline 23 & .6346446 & -0.4685362 & -0.3888307 & 0.3922332 & .4746973 & .6424078 \\
\hline 24 & 90699 & -0.4470427 & 737693 & 876159 & 714896 & 6303872 \\
\hline 25 & .5969210 & -0.4403808 & -0.3640087 & 0.3738185 & .4486703 & .6117672 \\
\hline 26 & 948354 & -0.4333082 & 688962 & 573695 & 476189 & 6044995 \\
\hline 27 & .5664993 & -0.4242786 & -0.3523856 & 0.3612035 & 0.4326448 & .5724763 \\
\hline 28 & 519401 & 117771 & 414448 & 553037 & 225960 & 5677018 \\
\hline 29 & & & & & 174685 & \\
\hline 30 & 387873 & 69189 & 328127 & 0.3427461 & 111536 & .5479877 \\
\hline 31 & & & 62 & & & \\
\hline 32 & 032165 & 3816363 & 184235 & 286855 & 0.3973704 & 0.5340919 \\
\hline 33 & 472 & -0.375 & 3135198 & 55 & 973 & 329 \\
\hline 34 & .4960804 & -0.3708996 & -0.3096764 & 0.3144957 & 0.3827077 & 0.5090763 \\
\hline 35 & -0.4950426 & 3677209 & -0.3090020 & 0.3089990 & 780428 & .5074862 \\
\hline 36 & .4303312 & -0.3624304 & 3013255 & 0.3086409 & 739596 & .5034182 \\
\hline 37 & .4745468 & -0.3538984 & 961882 & 0.3042587 & 675779 & 0.4948321 \\
\hline 38 & .4681570 & 509527 & 928190 & 0.2991383 & 574701 & 0.4882042 \\
\hline 39 & -0.4619455 & -0.3448189 & 870099 & 0.2909621 & 0.3519658 & 0.4831272 \\
\hline 40 & .4470719 & -0.3378060 & -0.2829040 & 0.2855017 & 0.3439096 & 0.4807485 \\
\hline 41 & -0.4462656 & -0.3317441 & -0.2786154 & 0.2827952 & 0.3383323 & 0.4641474 \\
\hline 42 & -0.4371849 & -0.3287158 & -0.2760627 & 0.2788424 & 0.3339745 & 0.4473633 \\
\hline 43 & -0.4296181 & -0.3228238 & -0.2670903 & 0.2763385 & 0.3303542 & 0.4457682 \\
\hline 44 & -0.4255031 & & & & 0.3293325 & 0.4425773 \\
\hline 45 & -0.4164150 & -0.3158671 & -0.2653876 & 0.2702089 & 0.3257615 & 0.4380333 \\
\hline
\end{tabular}

